\title{
Routing in CNC Cutting Machines: Engineering Constraints
}

\author{
Alexander A. Petunin ${ }^{1,2}$, Pavel A. Chentsov ${ }^{2,1}$ \\ ${ }^{1}$ Ural Federal University, 19 Mira Str., 620002 Ekaterinburg, Russia, \\ a.a.petunin@urfu.ru \\ ${ }^{2}$ Krasovskii Institute of Mathematics and Mechanics (IMM UB RAS), 16 S. \\ Kovalevskaya Str., 620990 Ekaterinburg, Russia, chentsov.p@uran.ru
}

Abstract: The paper is devoted to the problems of cutting path optimization with engineering specifics. There are two severe constraints of thermal cutting: thermal expansion and sheet stiffness constraints. Our solution uses the dependence of the cost functions on the previous part of the route and on the direction of movement of the tool along the contour.

Keywords: general traveling salesman problem; cutting tool motion optimization; CNC cutting machines

\section{Introduction}

One of the main ways of metal processing is metal sheet cutting. The common problems of metal sheet cutting [1] represent serious practical interest. A detailed overview is given in paper [2]. The main problem is to get parts of the best quality with the minimal cost. The cost includes plant work time and used resources during cutting process (gas, electricity, etc.).

The common problems of metal sheet cutting (see [1]) consist of the nesting problem and searching of accessible piercing points and cutting path optimization (tool path problem). This work is devoted to the tool path problem and its special constraints. There are constraints for enclosure of contours, for thermal expansion and for sheet stiffness.

The tool path problem is a very complex problem. It unites discrete and continuous optimization. There is no mathematical formalization of the problem for the common case. In the paper [3] it was proposed the version of the problem as a generalized travelling salesman problem (GTSP) with the inclusion of contours. Work [4] has a similar problem statement, but another way of heuristic 
solution (Tabu search). In the paper [5] the modification of the problem for the Leather cut process is shown. So, the existing mathematical models and algorithms for solving the problem do not consider most of the real technological constraints of the cutting process. In particular, it concerns the constraints connected with thermal deformations of parts at thermal cutting. But only the class of problems which are reduced to the minimization of idling motion of the cutter are considered. Some technological constraints of the cutting process and solving of tool path problem are described in [6].

The tool path optimization problem can be reduced to GTSP by adding new constraints and adding contour visit parameters. The constraints are precedence conditions, thermal and rigidity constraints. The tool motion direction is a parameter.

There are versions of GTSP with fixed permitted motions from point to point and the exact branch-and-cut algorithm and heuristic algorithm are given in [7].

The exact dynamic programming-based algorithm for a routing problem depending on the list of tasks' costs was proposed in [8]. The dinamyc programming fitted for CNC cutting machine tool routing is shown in [9].

The basic theory of tool routing optimization for CNC cutting machines is given in [10], the heuristic algorithm of problem solution is proposed in [11].

In [12] there are proposed heuristic methods of GTSP solving. It is a genetic algorithm with local improvement of solution. The same process was used in [13].

In the paper [14] we can see a hard metric heuristic algorithm and significant comparison with existing algorithms. The comparison shows the high efficiency of the algorithm.

This work is a continuation of [15]. The main difference is cost functions with using of rigidity constraint by penalties.

\section{Mathematical Formulation}

The steel sheet of fixed size and planned within it contours of the cutting details are given. By $p^{s t}$ and $p^{\text {fin }}$ denote a start and finish points of the cutting tool motion ( $p^{s t} \in R \times R, p^{\text {fin }} \in R \times R, R$ - real line). $N$ - number of contours. $V_{\text {idling }}$ - idling speed of the tool. $V_{c u t}$ - work speed. The piercing points must be realized out of the detail contour curves, because it leads to strong temperature expansion. Moreover, the hole in the point of insert can exceed cut width. Point $p^{o n}, p^{o n} \in R \times R$, is a piercing point. The cutting tool moves on work speed 
$V_{c u t}$ to the point of the enter in the contour $p^{c u t}, p^{c u t} \in R \times R$, and performs cut of the contour with finish in the point $p^{\text {cut }}$. The cut can be made clockwise or counter clockwise. A cutting tool switch-off can damage a detail edge, and cutting tool moves on work speed to the cut-off point $p^{\text {off }}, p^{\text {off }} \in R \times R$. The point $p^{o f f}$ placed out of the contour curve. In the point $p^{\text {off }}$ the cutting tool realizes switch-off, then starts motion on idling speed to the next contour. These points are shown on Figure 1.

There are thermal restrictions, requiring that near the finishing line of the contour cut there must be enough metal. It's shown in Figure 2.

The rigidity restriction, requiring that around large parts must be enough metal. It's shown in Figure 3.

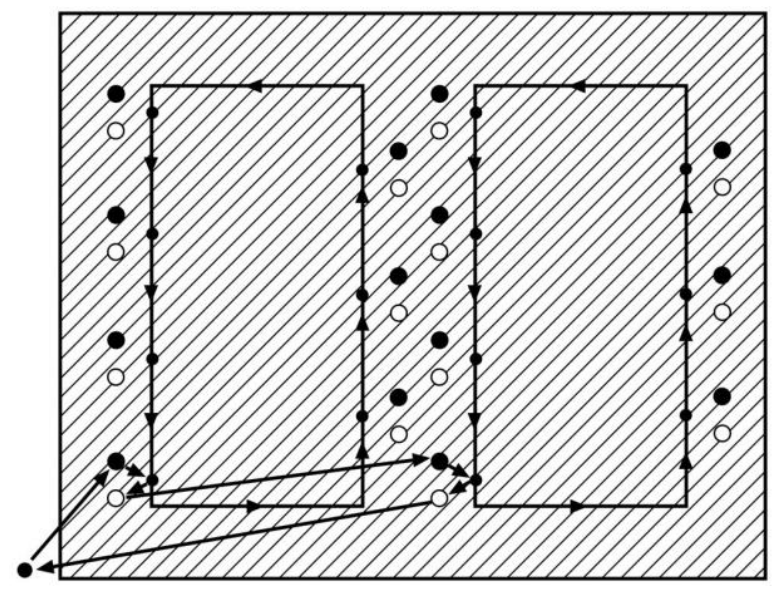

Figure 1

The cut points
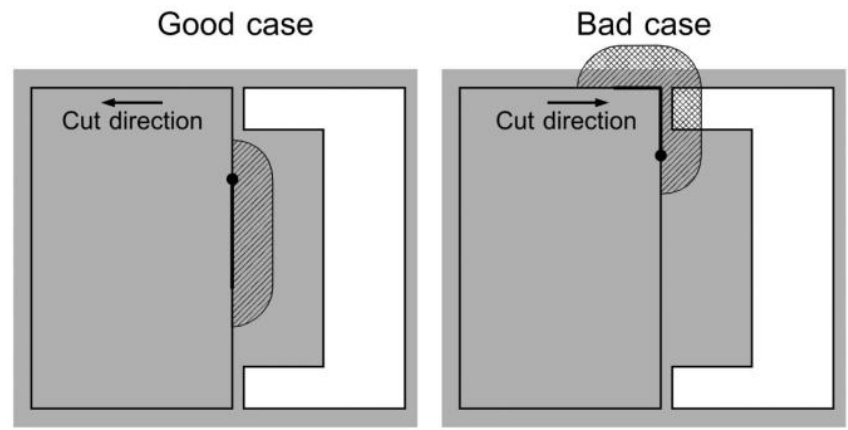

Figure 2

Thermal constraints 


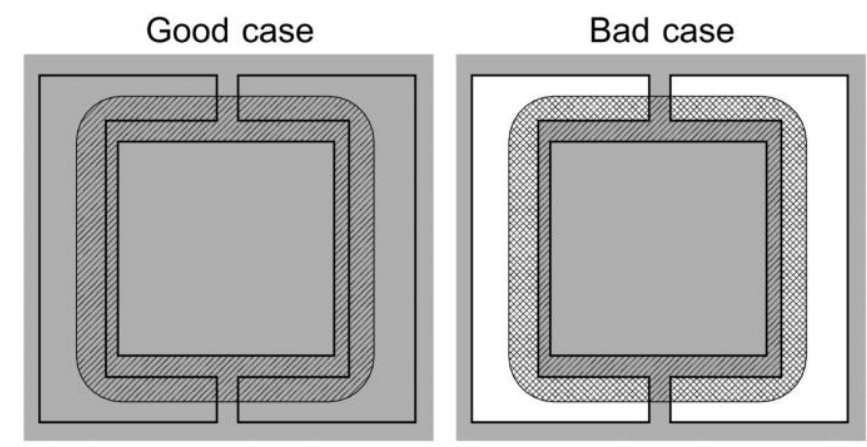

Figure 3

Rigidity constraints

Every contour cut begins and ends from the point triplet $-p^{o n}, p^{\text {cut }}, p^{o f f}$. The number of these triplets is $\tilde{N}$. Set $M, M=\overline{1, \tilde{N}}$, is the set of all triplet indexes. Let $M_{1}, \ldots, M_{N}, N \geq 2$, be sets of the contour point triplet indexes for contours. Each contour has its own number of the cut point triplets: $\left|M_{i}\right|=n_{i}$, $n_{i} \geq 1$. Note that

$$
\bigcup_{i \in 1, N} M_{i}=M, M_{i} \cap M_{j}=\varnothing \forall i \in \overline{1, N}, \forall j \in \overline{1, N} \backslash\{i\} .
$$

For the definition of the cut points denote three functions:

$$
\begin{aligned}
& p^{\text {on }}: M \rightarrow R \times R, \\
& p^{\text {cut }}: M \rightarrow R \times R, \\
& p^{\text {off }}: M \rightarrow R \times R .
\end{aligned}
$$

By $A$ we denote the family of the ordered sets. If $a \in A$, then

$$
\begin{aligned}
& a=\left(a_{i}\right)_{i \in 1, N}: \overline{1, N} \rightarrow M, \forall a_{1} \in a, \forall a_{2} \in a \backslash\left\{a_{1}\right\}, \neg \exists i \in \overline{1, N}: \\
& \left(a_{1} \in M_{i}\right) \&\left(a_{2} \in M_{i}\right)
\end{aligned}
$$

For $a \in A$ the route of cutting tool motion is

$$
\begin{aligned}
& p^{s t} \rightarrow p^{o n}\left(a_{1}\right) \rightarrow p^{\text {cut }}\left(a_{1}\right) \rightarrow p^{o f f}\left(a_{1}\right) \rightarrow \ldots \\
& \ldots \rightarrow p^{\text {on }}\left(a_{N}\right) \rightarrow p^{\text {cut }}\left(a_{N}\right) \rightarrow p^{o f f}\left(a_{N}\right) \rightarrow p^{f i n}
\end{aligned}
$$


For the definition of the internal cost (thermal restriction penalties), we need the direction sign $d, d \in\{0,1\}$. Value 0 corresponds to counter-clockwise contour cut process, 1 - clockwise.

By $D$ denoting the ordered set of contour tool motion directions for route: if $d \in D$, then $d=\left(d_{i}\right)_{i \in 1, N}: \overline{1, N} \rightarrow\{0 ; 1\}$.

If $d_{i}=1$, then cut of the contour cut in route position $i$ direction is clockwise, if $d_{i}=0$ - counter clockwise.

The speeds of the tool are significant parameters used in the formulas of cost functions. By $V_{\text {idling }}$ denoting idling speed. By $V_{\text {cut }}$ denoting work speed. An estimation of the idling motion from point to point is the motion time. If $x_{1}$ and $x_{2}$ are start and finish points of the switched-off cutting tool local motion ( $p^{s t}, p^{f i n}, p^{\text {on }}$ or $p^{\text {off }}$ ), then this motion can be evaluated by

$$
C\left(x_{1}, x_{2}\right)=\frac{\rho\left(x_{1}, x_{2}\right)}{V_{\text {idling }}} \text {. }
$$

In this formula $\rho$ is Euclidean distance between points $x_{1}$ and $x_{2}$.

An estimation of contour $i, i \in \overline{1, N}$, cutting with cut points triplet $m, m \in M_{i}$ and direction $d$ and cut out at this time contours set $K, K \subset \overline{1, N} /\{i\}$ :

$$
\begin{aligned}
& c(m, d, K)=\frac{\rho\left(p^{o n}(m), p^{c u t}(m)\right)}{V_{c u t}}+\frac{\rho\left(p^{c u t}(m), p^{o f f}(m)\right)}{V_{c u t}}+ \\
& c_{d}(m, d, K)+\widetilde{c}_{d}(m, K) .
\end{aligned}
$$

Contour cut time is excluded from the estimation because it must be made once, and does not depend on route.

Function $c_{d}$ is penalty function for cut finishing by location of finish cut area with respect to holes in metal from cut out contours at this time. Note that for final result estimation $c_{d} \equiv 0$, it is needed only when counting.

Function $c_{d}$ is defined as follows:

$$
c_{d}(m, d, K)=\frac{S_{m}^{*}(m, d, K)}{S_{m}(m, d, K)} P
$$


$S_{m}(m, d, K)$ - square of checking area near the finishing line of contour $i, i \in \overline{1, N}$, if cut makes with using triplet $m \in M_{i}$. Checking area near the finishing line of contour further will be referred as cut finish area. $S_{m}^{*}(m, d, K)-$ square of intersection area of cut finish area with cut out parts or out of sheet space (for detail see figure 2); $P$ - penalty coefficient. The method of $S_{m}$ and $S_{m}^{*}$ computation is shown in [17].

Function $\tilde{c}_{d}$ is penalty function for spaces around parts (rigidity constraint). It is meaningful for the large and heavy parts. So, as it shown in Figure 3, large parts must have enough metal around they external contours. If $m \in M_{i}$, then function $\tilde{c}_{d}$ is defined this way:

$\widetilde{c}_{d}(m, K)=\sum_{j \in \overline{1, N \backslash(K \cup\{i\})}} \widetilde{P}_{1}\left(\frac{\hat{S}(j)}{\widetilde{P}_{2}}\right)^{2} \times\left(1-\frac{\widetilde{S}^{*}(j, K \cup\{j\})}{\widetilde{S}(j)}\right) \times T(j)$

$\hat{S}(j)$ - square of metal within contour with index $j, j \in \overline{1, N}$ (without square of internal contours). $\tilde{P}_{1}$ - rigidity constraint weight coefficient. $\widetilde{P}_{2}$ - coefficient of part weight checking. $\tilde{S}(j)$ - square of checking external space near contour with index $j, j \in \overline{1, N}$ (further external contour area). $\tilde{S}^{*}(j, K)$ - square of external contour area for contour $j, j \in \overline{1, N}$, intersection with cut out contours and out of sheet space.

$T(j)$ for contour $j, j \in \overline{1, N}$, has value 1 if contour $j$ is the external contour of the part, and 0 otherwise.

In every step of route construction, $\tilde{c}_{d}(m, K)$ gives information on the correctness of the arrangement of the remaining contours with respect to the spaces in the metal and the space outside the sheet.

Note that for final result estimation, $\tilde{c}_{d} \equiv 0$, it is needed only when count as in case of $c_{d}$ function.

This problem has precedence conditions. In this paper a complete cutting of each contour is considered. Cut out contours drop down and became inaccessible to any further processing. In some situations cut out parts have support from below and stay accessible to processing. But they lose connection with the sheet and can have unaccounted by plant displacement. This leads to inaccuracies in the cut. So, the reason for the precedence conditions - internal contours must be cut out before external. 
It should be said that if we will consider situations with partial contour cut, it allows for making the cut of the internal contours after the partial cut of the external contours.

Let $Z$ be the set of address pairs $z$ for precedence condition:

$$
z=\left(z_{1}, z_{2}\right), z_{1} \in \overline{1, N}, z_{2} \in \overline{1, N}, z_{1} \neq z_{2}, Z \mid \geq 0 \text {. }
$$

For $z \in Z$ contour with index $z_{1}$ must be visited before contour with index $z_{2}$.

The set $Z$ makes some routes from $A$ inaccessible. We need to define the family of accessible routes $\tilde{A}, \tilde{A} \subset A: \forall a \in \tilde{A}, \forall z \in Z$ if $a_{i} \in M_{z_{1}}$ and $a_{j} \in M_{z_{2}}$ then $i<j$.

If $a \in \widetilde{A}$ and $d \in D$, then it's estimation is

$$
\begin{aligned}
& T(a, d)=C\left(p^{s t}, p^{o n}\left(a_{1}\right)\right)+c\left(a_{1}, d_{1}, \varnothing\right)+ \\
& \sum_{i=2}^{N}\left(C\left(p^{o f f}\left(a_{i-1}\right), p^{o n}\left(a_{i}\right)\right)+c\left(a_{i}, d_{i}, \bigcup_{j \in 1, i-1} a_{j}\right)\right)+C\left(p^{o f f}\left(a_{N}\right), p^{f i n}\right) .
\end{aligned}
$$

The main task is

$$
T(a, d) \rightarrow \min , a \in \tilde{A}, d \in D .
$$

\section{Computation of Area Squares for Rigidity Constraint}

The way of $S_{m}$ and $S_{m}^{*}$ computation for thermal constraints is shown in [17]. It is made by using special area matrixes in not an exact way but made permissible for this problem's precision.

The same method is used for $\hat{S}(j), \widetilde{S}(j)$ and $\widetilde{S}^{*}(j, K)$ computation.

The steel sheet and space around the sides splits in square areas - it is matrix cells. If coordinates of the cell are located out of the sheet, it links with value -2 . If the cell locates out of any contours, but within the steel sheet, it links with value -1 . If the cell intersects with some contour or locates within it, this cell links with this contour index (see Figure 4). 


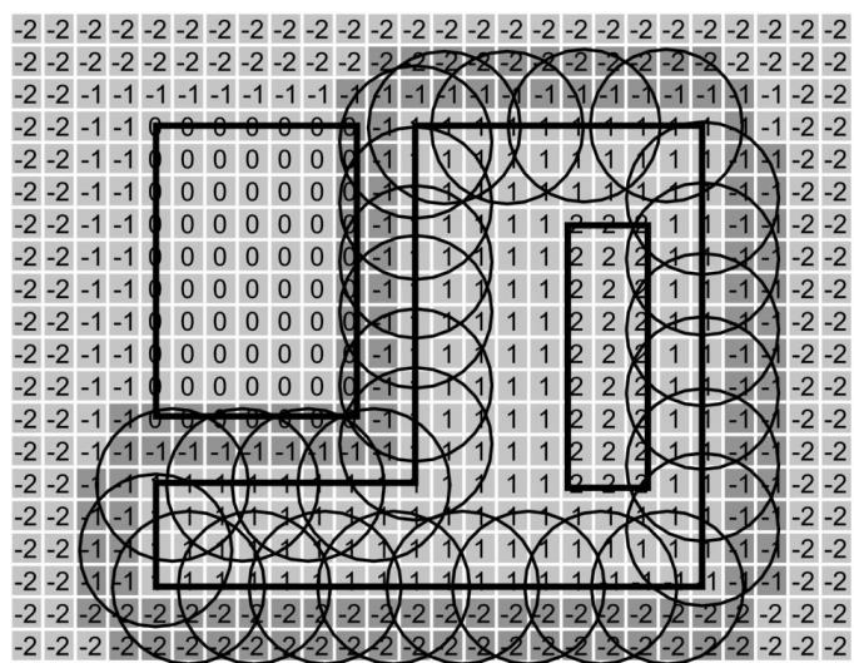

Figure 4

Rigidity area squares count

Count of the $\hat{S}(j)$ makes this way: for the contour with index $j, j \in \overline{1, N}$ we need to count the number of cells with value $j$, and multiply it by the cell square.

The following describes the method of $\widetilde{S}(j)$ computation for the contour with index $j, j \in \overline{1, N}$. Locate $N_{L}$ points on the contour on an equal range. Make a circular area around every point, and check intersections with area matrix cells. Calculate the number of cells, which value differs from $i$ (in Figure 4 it is 1) and indexes of its nested contours (in Figure 4 it is 2). Every cell must be checked once. Multiply this value by square of the cell. It gives $\widetilde{S}(j)$.

The following describes the method of $\widetilde{S}^{*}(j, K)$ computation for the contour with index $j, j \in \overline{1, N}$, and cut out at this time contours set $K, K \subset \overline{1, N}$. Locate $N_{L}$ points on the contour on equal range. Make a circular area around every point, and check intersections with the area matrix cells. Calculate the number of cells, which value is -2 or exists in the set $K$ and not an internal contour of contour with index $j$. Every cell must be checked once. Multiply this value by the square of cell. It gives $\widetilde{S}^{*}(j, K)$. 


\section{Algorithms for Problem Decision}

To solve problem (3) use exact dynamic programming (DP) method based algorithm from [9]. The heuristic algorithm of problem solving was proposed in [17], and is usable in this case too. This algorithm can be used with the new rigidity constraint because this constraint is implemented by penalty (2) in cost function (1), and does not make any improvements directly in the algorithms.

The exact method form [9] can be used for small samples with the number of contours less then 27. The heuristic algorithm allows for obtaining route for hundreds of contours. It is usable in most real industrial cuttings.

\section{Computing Experiments}

Calculations were made on the computer with the Intel i7-2630QM processor, 8 GB memory and operating system Windows 7 (64-bit).

The program is developed with using $\mathrm{C}++$, in the development environment Microsoft Visual Studio 2013.

The samples contain parts of different sizes. It allows for showing the work of algorithms with using new cost functions.

Full information on coordinates of contours, cut points, and cut sequence is not included due to space economy reasons. Even for the first small sample, it requires a lot of text pages;

The external contour area width value is $200 \mathrm{~mm}$. This width seems to be enough for making good rigidity for most large parts, but the search of the most appropriate value of this parameter can be a topic for the new research. Coefficient values: $\widetilde{P}_{1}=1, \widetilde{P}_{2}=1000000$. These parameters were used for all samples.

Example 1. Parameters: $N=16,|Z|=4$.

The first count was made with DP exact method with a counting duration of 21 seconds. Value of estimation is 64,82. The route is shown in Figure 5. 


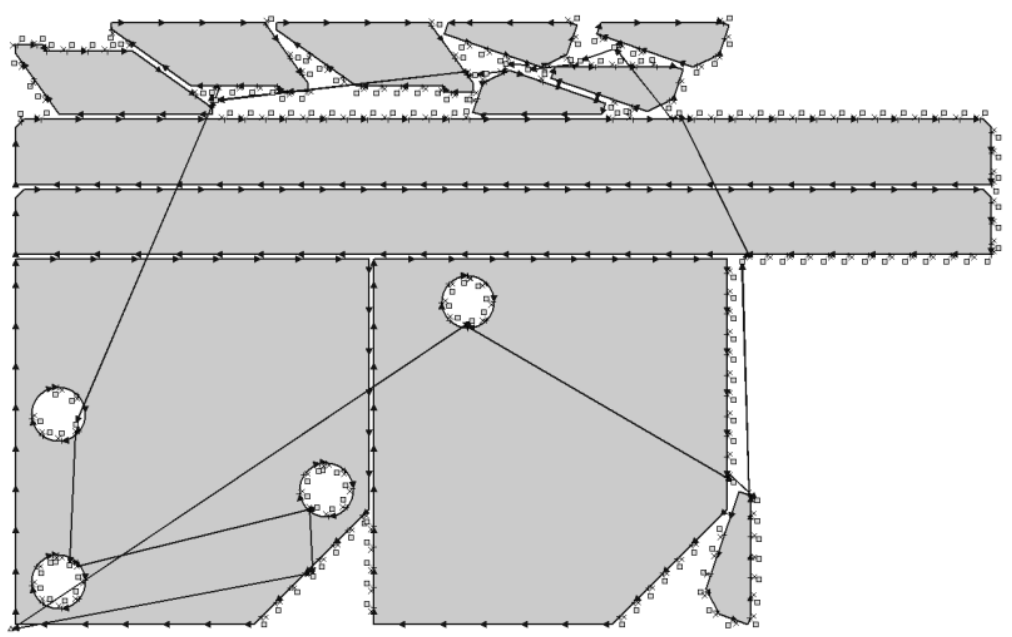

Figure 5

Result for DP count, sample 1

Second count was made with heuristic method. Counting duration 4 seconds. Value of estimation 66,88. The route is shown on Figure 6.

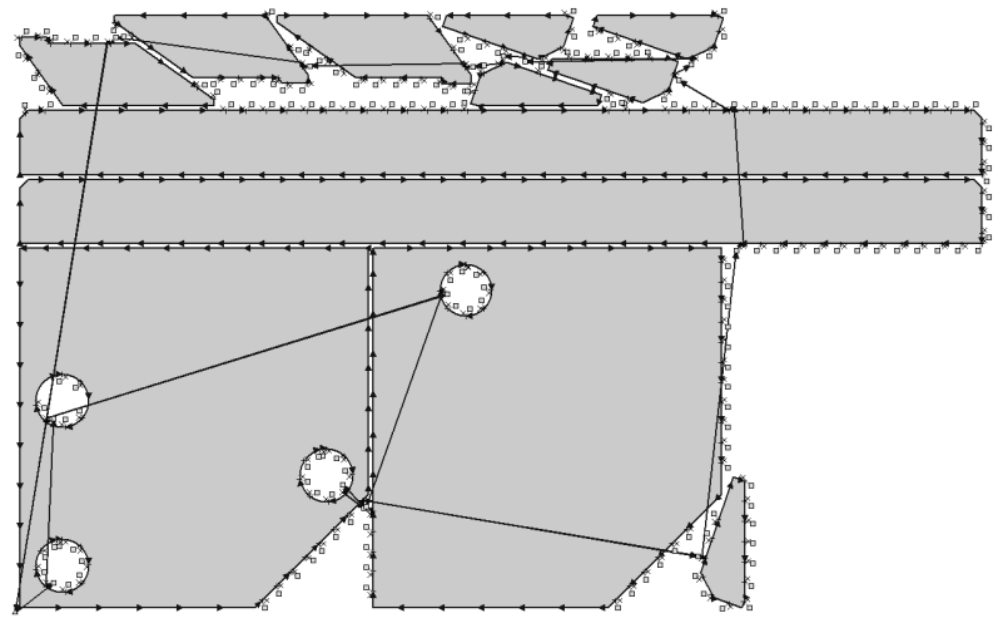

Figure 6

Result for heuristic count, sample 1

Example 2. Parameters: $N=86,|Z|=29$. To obtain decision the heuristic method (to many contours for DP) was used. The counting duration was 13 seconds. For computation aiterative process was used with a total iterations count of 10. If count time seems to long, it can be reduced to a lower value (less then 10). Value of estimation was 295,33 . The route is shown on Figure 7. 


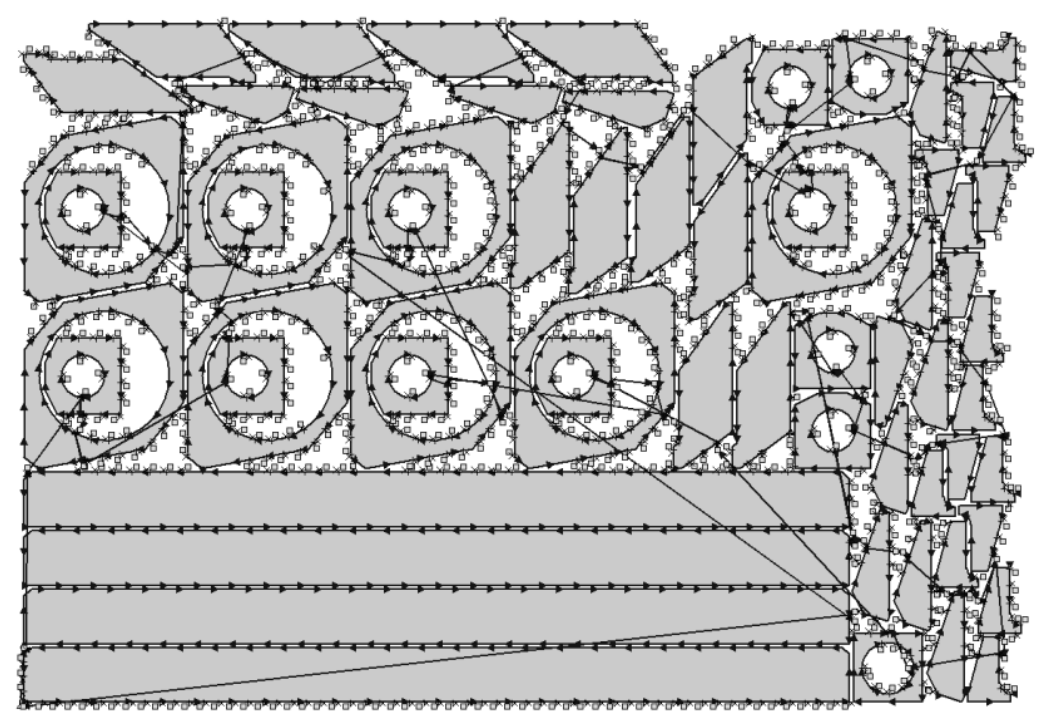

Figure 7

Result for heuristic count, sample 2

So, we can see that the performance of the algorithm allows for calculation of tasks of large dimensions. Is makes the algorithm applicable in manufacturing.

The heuristic algorithm does not gives an exact decision, but the formulation of problems is based on our assumptions about the quality of the cut. We take coefficient and parameter values by our mention on the basis of our ideas about the physical process. This assumptions can differ with real physical process features. In this situation the exact decision of the formulated tasks can be difficult to obtain real cut quality. This circumstance makes the application of exact algorithms not so important, although desirable.

\section{Conclusions}

Some problems of tool routing for $\mathrm{CNC}$ sheet cutting machines are considered. Complex specific engineering constraints are constructed. The method of constraints computation is proposed.

New constraint is fitted to use with existing exact and heuristic algorithms of tool path optimization problem.

Several computing experiments were performed. The resulting routes look good in respect to constraints.

\section{Acknowledgement}

This work was supported by Russian Foundation for Basic Research, grant 20-0800873. 


\section{References}

[1] A. A. Petunin: Development of CAM-system for sheet cutting machines as an innovation example, Innovative information technologies: Theory and practice. International scientific edition: materials of the International workshop (Karlsruhe - Ufa - Dresden, April 8-13, 2011), 2011, Ufa, pp. 47-50

[2] Petunin A. A., Stylios C. Optimization models of tool path problem for CNC sheet metal cutting machines. IFAC-PapersOnLine. - 2016 - V. 49, 1. 12. - pp. $23-28$

[3] K. Castelino, R. D'Souza, P: Wright Tool-path optimization for minimizing airtime during machining, Journal of Manufacturing Systems 22 (3), 2002, pp. $173-180$

[4] Dewil, R., Vansteenwegen, P., Cattrysse, D. (2016) A review of cutting path algorithms for laser cutters. Int J Adv. Manuf. Technol. 87:1865-1884

[5] W. B. Yang, Y. W. Zhao, J. Jie, W. L. Wang: An Effective Algorithm for Tool-Path Airtime, Optimization during Leather Cutting. Advanced Materials Research. 2010, 102-104, pp. 373-377

[6] Petunin, A. (2009) About some strategies of the tool path modeling at the control programs generation for the flame cutting machines. Vestnik UGATU, Vol. 13, 2(35), 280-286

[7] M. Fischetti, J. J. Salazar-Gonzalez, P. Toth: A Branch-and-Cut algorithm for the symmetric generalized traveling salesman problem: Operations Research. 1997, 45 (3), pp. 378-394

[8] Chentsov, A. G., Chentsov, A. A. Dynamic programming in the routing problem with constraints and costs depending on a list of tasks. Dokl. Math. 2013, 88, 637-640

[9] A. G. Chentsov, P. A. Chentsov, A. A. Petunin, A. N. Sesekin: Routing problems: constraints and optimality, IFAC-PapersOnLine. 2016, Vol. 49, Issue 12 , pp. 640-644

[10] Chentsov, P. A. About one application of greedy algorithm for the solution of the generalized salesman problem. Proceedings of the 16h International Workshop on Computer Science and Information Technologies. Sheffield, England, 2014, Vol. 2, pp.148-151

[11] A. A. Petunin, A. G. Chentsov, P. A. Chentsov: About routing of tool motion in the sheet cutting machines with numerical program control, Scientific and technical sheets SPbGPU. Informatics. Telecommunications. Management, 2013, 2 (169), pp. 103-111

[12] L. V. Snyder, M. S. Daskin: A random-key genetic algorithm for the generalized traveling salesman problem, European Journal of Operational Research, 2006, 174, pp. 38-53 
[13] J. Silberholz, B. Golden: The Generalized Traveling Salesman Problem: a new Genetic Algorithm approach. Extending the Horizons: Advances in Computing, Optimization, and Decision Technologies, 2007, 37, pp. 165181

[14] G. Gutin, D. Karapetyan. A Memetic: Algorithm for the Generalized Traveling Salesman Problem, Natural Computing, 2010, 9(1), Springer, pp. 47-60

[15] P. A. Chentsov, A. A. Petunin: Tool Routing Problem for CNC Plate Cutting Machines, IFAC-PapersOnLine. 2016, Vol. 49, Issue 12, pp. 645650 